Association for Information Systems AIS Electronic Library (AISeL)

Wirtschaftsinformatik Proceedings 2003

Wirtschaftsinformatik

September 2003

\title{
DART - Ein Ansatz zur Analyse und Evaluierung der Benutzerakzeptanz
}

Michael Amberg

Friedrich-Alexander-Universität Erlangen-Nürnberg

Markus Hirschmeier

Friedrich-Alexander-Universität Erlangen-Nürnberg

Deniz Schobert

Friedrich-Alexander-Universität Erlangen-Nürnberg, Deniz.Schobert@wiso.uni-erlangen.de

Follow this and additional works at: http://aisel.aisnet.org/wi2003

\section{Recommended Citation}

Amberg, Michael; Hirschmeier, Markus; and Schobert, Deniz, "DART - Ein Ansatz zur Analyse und Evaluierung der Benutzerakzeptanz" (2003). Wirtschaftsinformatik Proceedings 2003. 30.

http://aisel.aisnet.org/wi2003/30

This material is brought to you by the Wirtschaftsinformatik at AIS Electronic Library (AISeL). It has been accepted for inclusion in Wirtschaftsinformatik Proceedings 2003 by an authorized administrator of AIS Electronic Library (AISeL). For more information, please contact elibrary@aisnet.org. 
In: Uhr, Wolfgang, Esswein, Werner \& Schoop, Eric (Hg.) 2003. Wirtschaftsinformatik 2003: Medien - Märkte - Mobilität, 2 Bde. Heidelberg: Physica-Verlag

ISBN: 3-7908-0111-9 (Band 1)

ISBN: 3-7908-0116-X (Band 2)

(C) Physica-Verlag Heidelberg 2003 


\title{
DART - Ein Ansatz zur Analyse und Evaluierung der Benutzerakzeptanz
}

\author{
Michael Amberg, Markus Hirschmeier, Deniz Schobert \\ Friedrich-Alexander-Universität Erlangen-Nürnberg
}

\begin{abstract}
Zusammenfassung: Die Benutzerakzeptanz wird zunehmend als kritischer Faktor für die Entwicklung innovativer Anwendungen und deren Markterfolg angesehen. Das vorliegende Paper stellt ein Akzeptanzmodell vor, das im Sinne eines allgemeinen Bewertungsinstruments einen Beitrag zur Analyse und zum Design innovativer Anwendungen leistet. Das als DART-Ansatz bezeichnete Bewertungsinstrument basiert auf der grundlegenden Idee der Balanced Scorecard. Demnach wird zur Bewertung der Benutzerakzeptanz ein ausgewogenes Verhältnis von individuell messbaren Akzeptanzkriterien verwendet. Ausgerichtet auf die jeweilige Anwendung werden die relevanten Einflussfaktoren systematisch identifiziert, unabhängig davon, ob sie direkt oder indirekt, rational oder emotional auf die Benutzerakzeptanz wirken. Die ausgewogene Betrachtung von mehreren akzeptanzrelevanten Kriterien führt zu einer differenzierten Bewertung der Benutzerakzeptanz. Der DART-Ansatz kann als stetiges Steuerungsinstrument für die wiederholte Anpassung einer Anwendung an die Bedürfnisse der Anwender aufgefasst werden.
\end{abstract}

Schlüsselworte: Akzeptanzforschung, Akzeptanzmodell, Balanced Scorecard, Bewertung von Innovationen, Innovative Anwendungen, Mobile Anwendungen

\section{Motivation}

Der Erfolg von Produkten und Dienstleistungen hängt meist unmittelbar von der Benutzerakzeptanz ab. Nur wenn der Benutzer ein Produkt bzw. eine Dienstleistung annimmt und nutzt, kann sie sich erfolgreich auf dem Markt positionieren. Dieses Erfolgskriterium trifft insbesondere auf Innovationen zu, da deren Nutzen oft nicht unmittelbar erkennbar ist. Als innovative Anwendung bezeichnen wir hier Softwareprodukte und softwarenahe Dienstleistungen (Datendienste), die im weitesten Sinne eine für den Benutzer subjektiv empfundene Neuerung zu den bereits am Markt etablierten Produkten bieten. Dabei stellen häufig neue Technologien die Basis von Innovationen dar, die zu innovativen Anwendungen führen.

Die Benutzerakzeptanz wird zunehmend nicht nur durch rationale Faktoren wie Funktionalität, Einfachheit in der Handhabung oder Kosten bestimmt. Vielmehr lässt sich ein Trend erkennen, der auch ,weichen“ Faktoren wie Technologieaffinität, Partizipation an gesellschaftlichen Modeerscheinungen oder Skepsis vor 
Umstrukturierung und Erneuerungen eine Bedeutung zuspricht. Zur Veranschaulichung sollen folgende Beispiele dienen: Obwohl manche innovativen Anwendungen einen hohen Nutzen versprechen, beispielsweise durch die Erhöhung des Benutzerkomforts, Senkung der Betriebskosten oder Erhöhung der relativen Sicherheit, werden sie von den potenziellen Benutzern nicht angenommen (Beispiel biometrische Identifikationssysteme). Dahingegen gibt es Anwendungen, deren übermäßige Akzeptanz aus rationalen Gesichtspunkten nicht ohne weiteres vorauszusehen war (Beispiel Short Message Service).

Um die erfolgskritischen Hindernisse zu minimieren, ist eine umfangreiche und gründliche Betrachtung der Einflussfaktoren auf die Benutzerakzeptanz hilfreich. $\mathrm{Zu}$ diesem Zweck haben in der Vergangenheit Wissenschaftler verschiedener Disziplinen so genannte Akzeptanzmodelle entwickelt, die einen Beitrag zur Erklärung der Benutzerakzeptanz leisten. Das vorliegende Paper führt in die Akzeptanzforschung ein und stellt relevante Akzeptanzmodelle vor. Diese Modelle sind nicht darauf ausgerichtet, die gewonnenen Erkenntnisse in die Neuentwicklung bzw. Weiterentwicklung einer Anwendung einfließen zu lassen, um so Produkte zu entwickeln, die den Erwartungen der Benutzer gerecht werden. Die Zielsetzung des DART-Ansatzes ist es, dieses Defizit zu verringern, indem er unmittelbar auf die Unterstützung der Anwendungsentwicklung ausgerichtet ist und zudem die spezifischen Anforderungen innovativer Anwendungen bei der Analyse und Evaluierung der Benutzerakzeptanz berücksichtigt. Seine Methodik und sein Vorgehen werden in den Folgekapiteln beschrieben.

\section{Einführung in die Akzeptanzforschung}

Das Phänomen der Akzeptanz ist Gegenstand zahlreicher wissenschaftlicher Untersuchungen. Aufgrund der unterschiedlichen Sichtweisen und Intepretationen besteht eine Vielzahl nicht-einheitlicher Definitionen. Der Begriff Akzeptanz wird im allgemeinen Verständnis von Simon als „Widerspruch zum Begriff Ablehnung definiert und bezeichnet die positive Annahmeentscheidung einer Innovation durch den Anwender" [Sim01, 87]. Der Untersuchungsgegenstand der Akzeptanzforschung konzentriert sich dabei auf zwei Zielsetzungen:

- Analytische Zielsetzung: Erklärung der Wechselbeziehungen zwischen der Einführung von Innovationen und ihren Auswirkungen.

- Gestaltende Zielsetzung: Beeinflussung der Ausgestaltung von Innovationen im Hinblick auf ihre Nutzung durch den Anwender.

Die Akzeptanzforschung findet ihren Ursprung sowohl in der Arbeitswissenschaft als auch in der Betriebswirtschaftslehre. Die arbeitswissenschaftlichen Betrachtungen fokussieren auf die Bedingungen bedienerfreundlicher Technikgestaltung. In der Betriebswirtschaftslehre wird die Akzeptanz in verschiedenen Disziplinen 
diskutiert und untersucht. Dazu gehören vor allem die Marketinglehre, die Organisationslehre, die Produktionstheorie und die Wirtschaftsinformatik [Döhl83, 110; Schr01, 64; Sim01, 86]. Während sich die Marketinglehre mit den Bedingungen der Annahme von Produkten durch den Markt befasst, untersucht die Organisationslehre die Bedingungen benutzeradäquater Gestaltung von Informationstechnologien. Die Produktionstheorie konzentriert sich auf die Untersuchung der Bedingungen der Input-Output-Zusammenhänge bei der einzelwirtschaftlichen Leistungserstellung [Döhl83, 111]. Der Untersuchungsgegenstand der Benutzerakzeptanz im Rahmen einer wirtschaftsinformatischen Betrachtungsweise bezieht sich auf die Akzeptanz von Informationssystemen (IS). Die Wirtschaftsinformatik konzentriert sich einerseits auf die Untersuchung von der Annahme innovativer Produkte sowie Dienstleistungen und andererseits auf organisatorische Maßnahmen, die mit der Einführung von IS verbunden sind. Sie befasst sich sowohl mit der analytischen als auch mit der gestaltende Zielsetzung.

Die isolierte Sichtweise der verschiedenen Diziplinen hat in der Vergangenheit zu einer umfassenden Anzahl von Theorien und Modellen zur Erklärung der Akzeptanz geführt. Der Versuch, Benutzerakzeptanz umfassend zu erklären, erfolgt meist in der Erarbeitung eines Akzeptanzmodells, mit dessen Hilfe der Benutzer und die Innovation miteinander in Beziehung gesetzt werden [Sim01, 86].

In der Akzeptanzforschung lässt sich eine Weiterentwicklung von den Forschungstraditionen hin zu einem neuen Verständnis der Akzeptanzforschung erkennen (neuere Akzeptanzforschung) [Koll98, Koll99b]. Vor diesem Hintergrund werden ausgewählte Merkmale vorgestellt, die den Trend in der Akzeptanzforschung der letzten Jahre geprägt haben:

- Betrachtungshorizont: Traditionell ist die Akzeptanzforschung Untersuchungsgegenstand von verschiedenen Wissenschaftsbereichen, die Akzeptanz isoliert voneinander betrachten. Diese stringente Einteilung ist heutzutage in Bezug auf innovative Anwendungen nicht mehr haltbar, da sich die meisten Technologien sowohl in einer Organisation (einem Unternehmen, einer gemeinnützigen Anstalt, einem Verein u.ä.) als auch im privaten Haushalt etablieren. Die isolierte Betrachtung der Akzeptanz und die dadurch entstandene Fokussierung auf besondere Aspekte führte in der Vergangenheit dazu, dass in jedem Bereich Schwachstellen auftraten [Döhl83, 111]. Im Gegensatz dazu setzt eine integrative Sichtweise die kritischen Erfolgsfaktoren der einzelnen Disziplinen zusammen.

- Entscheidungsmerkmal: Die Dichotomisierung (Ja/Nein-Entscheidung) der Akzeptanzentscheidung in der klassischen Akzeptanzforschung kann nicht grundsätzlich auf innovative Anwendungen übertragen werden. Insbesondere bei Nutzungsinnovationen, bei denen primär die Nutzungsintensität über den Markterfolg entscheidet, muss ein Akzeptanzkontinuum anerkannt werden, das Abstufungen zwischen hoher Akzeptanz (tendenziell hohe Nutzungshäufig- 
keit/Zahlungsintensität) und niedriger Akzeptanz (tendenziell geringe Nutzungshäufigkeit/Zahlungsintensität) berücksichtigt [Koll99b, 128].

- Nutzungsmotivation: Da die Diffusion innovativer Produkte in zunehmenden Maße sowohl in Organisationen als auch in Privathaushalten erfolgt, ist eine strikte Trennung des organisationsbedingten Nutzungszwangs und des freiwilligen Einsatzes nicht mehr möglich. Produkte der Informationstechnologie wie Personal Computer, Notebook, Personal Digital Assistant, der Telekommunikation, insbesondere des Mobilfunks, des Multimedia-Bereiches usw. werden organisationsbedingt und freiwillig genutzt.

- Zielsetzung: Im traditionellen Verständnis wird die Akzeptanz ex-post betrachtet. Diese Ausrichtung dient dazu, im Nachhinein geeignete Vermarktungsstrategien für bereits eingeführte Produkte und Dienstleitungen zu finden (expost Betrachtung) [Koll99b, 129]. Für das Erkennen von Produktdefiziten noch vor der Markteinführung und das damit verbundene frühzeitige Eingreifen in den Entwicklungsprozess ist diese Betrachtungsweise nicht zielführend. Mit dem Versuch, die Akzeptanz bereits zu einem möglichst frühen Zeitpunkt zu analysieren, lässt sich auf die Gestaltung der Innovation Einfluss nehmen (exante Analyse). Demzufolge ist eine Akzeptanzanalyse bereits vor der Markteinführung (z.B. über Prototypen oder virtuelle Produkte) durchzuführen [Koll98, 59], ohne auf eine ex-post Betrachtung gänzlich zu verzichten.

Im Folgenden werden ausgewählte Akzeptanzmodelle vorgestellt.

\section{3 Übersicht über relevante Akzeptanzmodelle}

Im Zuge ihrer Untersuchungen haben verschiedene Wissenschaftler Theorien und Modelle entwickelt, mit denen die Benutzerakzeptanz beschrieben und erklärt werden kann. In jedem dieser Modelle werden unterschiedliche Faktoren bestimmt, die zur Erklärung der Benutzerakzeptanz herangezogen werden. Folgende Akzeptanzmodelle aus dem angloamerikanischen und deutschsprachigen Raum werden kurz vorgestellt:

- Akzeptanzmodell von Degenhardt (1986)

- Technologieakzeptanzmodell von Davis (1989)

- Task Technology Fit Model von Goodhue (1995)

- Dynamisches Akzeptanzmodell von Kollmann (1998)

- Vorgehensmodell von Herrmann et al. (1999)

Das Akzeptanzmodell von Degenhardt wurde zur Akzeptanzerforschung des Bildschirmtextes entwickelt [Dege86]. Ausgangspunkt ist die Überlegung, dass die 
Akzeptanz maßgeblich von der wahrgenommenen Nützlichkeit eines Systems abhängt. Ein System wird vom Nutzer dann als nützlich eingestuft, wenn die angebotenen Funktionen für die Erledigung seiner Aufgabe geeignet sind. Die Nützlichkeit des Systems wird durch drei Untersuchungsdimensionen beeinflusst: Aufgabencharakteristika (Wichtigkeit, Häufigkeit, Erledigungsalternativen), Systemkonfiguration (Aufgabenkompatibilität, Benutzerfreundlichkeit, Erlernbarkeit) und Benutzermerkmale (Fähigkeiten, Fertigkeiten, motivationale Variablen, soziales Umfeld). Degenhardt beschreibt, dass letztlich erst eine gleichzeitige Wahrnehmung von Nützlichkeit und Akzeptierbarkeit zu einer individuellen Akzeptanz des Systems führt. In seinem Modell werden keine Rückkopplungsmechanismen vorgestellt, die den Nutzungsaspekt der Innovation berücksichtigen [Sim01, 98].

Das Technologieakzeptanzmodell (TAM) von Davis ist im angloamerikanischen Raum eines der am weitesten verbreiteten Modelle [Sim01, 94]. Es bestimmt die Benutzerakzeptanz mithilfe von zwei Faktoren: perceived usefulness (wahrgenommener Nutzen) and perceived ease of use (wahrgenommene einfache Benutzbarkeit) [Dav86]. Den wahrgenommenen Nutzen definieren Davis et al. als "the prospective user's subjective probability that using a specific application system will increase his or her job performance within an organizational context", während die wahrgenommene einfache Benutzbarkeit "the degree to which the prospective user expects the target system to be free of effort" [Dav 89 , 985] bedeutet. Auf beide Einflussfaktoren wirken externe Stimuli. Je höher der Nutzen eines Systems sowie dessen einfache Bedienbarkeit dadurch empfunden wird, desto eher ist der Anwender dazu bereit, die Innovation zu nutzen [Sim01, 102]. Als kritisch an dem Technologieakzeptanzmodell werden vor allem zwei Aspekte betrachet: Zum Einen sind die gewählten Einflussfaktoren nicht ausreichend, um die Akzeptanz umfassend und vollständig zu analysieren. Von einigen Wissenschaftlern wurde zum Beispiel bemängelt, dass TAM keine sozialen Aspekte als Einflussgröße definiert [Dav86; Dav89; $\mathrm{Dav}^{+}$89; HuCo94; Mel90]. Zum Anderen konnte nachgewiesen werden, dass die wahrgenommene einfache Benutzbarkeit nur einen geringen Einfluss auf die Akzeptanz hat [GeSt00].

Das Task Technology Fit Model (TTFM), das auf Goodhue zurückgeht, dient der Bewertung von Informationssystemen durch den Anwender. Dadurch, dass die Einschätzung der Leistungsfähigkeit des Benutzers seine Akzeptanz beeinflusst, kann es zu den Akzeptanzmodellen hinzu gezählt werden [Sim01, 95]. Für die Bewertung von Informationssystemen werden drei Faktoren als entscheidend angesehen: die Aufgabe (engl. task), die Technologie (engl. technology) und das Individuum (engl. individual) [Good95, 1831]. Alle drei Faktoren beeinflussen die individuelle Einschätzung des Systems, mit dem eine bestimmte Aufgabe erledigt werden soll. Das TTFM dient im engeren Sinne nicht dazu, die Akzeptanz der Benutzer zu erklären, sondern ihre Bewertung über ein System zu erfassen. Dabei wird die Akzeptanz als Hauptfaktor für die Leistungsbewertung gesehen. Das Modell strebt eine ex-post Betrachtung von Systemen an, die dem organisationsbedingten Nutzungszwang unterliegen. 
Kollmann betont in seinen Arbeiten den Bedarf der neueren Akzeptanzforschung, konzentriert sich dabei aber auf die von ihm eingeführte Produktkategorie der Nutzungsinnovationen [Koll98, Koll99a, Koll99b]. Sein dynamisches Akzeptanzmodell basiert auf drei Akzeptanzebenen, die einen Hinweis auf den prozessualen und dynamischen Charakter der Akzeptanzbildung geben:

- Einstellungsphase: Eine Phase vor dem Kauf bzw. Übernahme einer Innovation

- Handlungsphase: Den Kauf- bzw. Übernahmezeitpunkt einer Innovation

- Nutzungsphase: Nach dem Kauf bzw. der Übernahme kommt die Innovation zum Einsatz

Mit dieser Einteilung wird erkennbar, dass es sich bei der Akzeptanz um eine dynamische Größe handelt. Die potenziellen Nutzer werden in drei Akzeptanztypen geteilt: Akzeptierer, Indifferente, Nicht-Akzeptierer. Kritisch an Kollmanns dynamischen Akzeptanzmodell sind vor allem zwei Aspekte, die aus der strikten Konzentration auf zwei Bereiche resultieren: Zum Einen betrachtet Kollmann ausschließlich die freiwillige Nutzung durch Privatanwender und zum Anderen leitet er seine Aussagen aus der Produktkategorie der Nutzungsinnovationen ab. Innovative Anwendungen, bei der die Nutzungsintensität nicht an Kosten gekoppelt ist, sind nicht Gegenstand seiner Betrachtungen.

Herrmann et al. konzentrieren sich im Rahmen ihrer Akzeptanzuntersuchungen auf den Mediensektor, insbesondere Mediendienste. Sie tragen verschiedene Bestimmungsgrößen, die auf die Nutzungsbereitschaft wirken, zusammen: Nutzerbedürfnisse und Nutzungsziel, subjektive Einstellungen, die durch das soziale Umfeld geprägt sind, Erwartungsdruck und soziale Normen, Restriktionen bzw. Ressourcen sowie Alternativen [Harn ${ }^{+} 99$ ]. Mit Hilfe einer Checkliste kann die Vielzahl und Vielfalt von Akzeptanzfaktoren strukturiert werden. Dabei unterscheidet sie drei Arten: übergreifende, individuelle (hier erfolgt eine separate Sichtweise zwischen Arbeitswelt und Konsumentenbereich) und gesellschaftliche Faktoren [HeMo99]. Eine der Besonderheiten dieser Checkliste ist die gemeinsame Betrachtung von Akzeptanz- und Kompetenzfaktoren der Benutzer. Aufbauend auf diesen Grundlagen entwickeln Herrmann et al. ein Vorgehensmodell zur Evaluierung von innovativen Anwendungen, das die verschiedenen Prozesse einer empirischen Erhebung in den Vordergrund stellt [Herr ${ }^{+} 99$ ]. Bei einer kritischen Würdigung des Modells von Herrmann et al. kann angemerkt werden, dass die empirische Erhebung stark in den Vordergrund gestellt wird. Infolgedessen fehlen Folgeaussagen zu den gewonnenen Erkenntnissen und ihren Konsequenzen. Des Weiteren wird die Rolle der Verantwortlichen für die einzelnen Prozesse nicht ausreichend heraus gearbeitet.

Im weitesten Sinne lassen sich Akzeptanzmodelle auch als eine Methode zur ständigen Qualitätsverbesserung eines Produktes interpretieren. Sie erlauben durch eine umfassende und ausgewogene Analyse und Evaluierung der Benutzerakzep- 
tanz, Defizite einer innovativen Anwendung zu identifizieren und die Auswirkungen ergriffener Gegenmaßnahmen zu messen. Mit diesen gewonnenen Kenntnissen lässt sich in den Produktentwicklungszyklus eingreifen, um das Produkt gegebenenfalls an die Erwartungen der (potenziellen) Benutzer anzupassen.

Da die bestehenden Ansätze diesen gestaltenden Aspekt bisher vernachlässigt haben, soll im Folgendem mit dem DART-Ansatz eine Methodik vorgestellt werden, welche unmittelbar darauf ausgerichtet ist, die Benutzerakzeptanz bei der Entwicklung innovativer Anwendungen zu berücksichtigen.

\section{Die Zielsetzung des DART-Ansatzes}

Der DART-Ansatz ist ein Instrumentarium, das auf die spezifischen Anforderungen der Analyse und Evaluierung der Benutzerakzeptanz von innovativen Anwendungen fokussiert. Das Akronym DART steht für Dynamisches Akzeptanzmodell zur Re-Evaluierung von Technologiebasierten Anwendungen. Der Begriff ReEvaluierung betont die Bedeutung einer wiederkehrenden Betrachtung der Benutzerakzeptanz.

Mit dem DART-Ansatz werden im Einzelnen folgende Zielsetzungen verfolgt:

- Verwendbarkeit als Bewertungsinstrument während des gesamten Produktlebenszyklus: Die Analyse und Evaluierung der Benutzerakzeptanz ist in allen Phasen eines Produktlebenszyklus sinnvoll. Während der Neuentwicklung einer Anwendung können entsprechende Erkenntnisse genutzt werden, um Stärken und Schwächen zu ermitteln, die Produktgestaltung zu beeinflussen oder Maßnahmen zur Erhöhung der Benutzerakzeptanz zu initiieren (ex-ante Analyse). Während der Nutzung einer Anwendung können die Erkenntnisse genutzt werden, um Hinweise für die Weiterentwicklung zu erhalten oder Maßnahmen zur Erhöhung der Benutzerakzeptanz zu ergreifen (ex-post Betrachtung).

- Einmalige und wiederholte Anwendbarkeit: Die einmalige Analyse und Evaluierung der Benutzerakzeptanz dient im wesentlichen der Standortbestimmung hinsichtlich der Stärken und Schwächen einer innovativen Anwendung. Die wiederholte Analyse und Evaluierung der Benutzerakzeptanz ermöglicht zusätzlich die Messung der Auswirkungen ergriffener Maßnahmen. Es wird dann sichtbar, welche Akzeptanzfaktoren inwieweit durch die Maßnahmen beeinflusst werden. Ein Akzeptanzmodell kann somit die Funktion eines Controllinginstruments übernehmen und zur Qualitätssicherung innovativer Anwendungen beitragen.

- Verwendbarkeit für Akzeptanzerhebungen im Arbeits- und Privatbereich: Im Sinne der neueren Akzeptanzforschung sind Akzeptanzmodelle sowohl aus 
Sicht des organisationsbedingten Nutzungszwangs in der Arbeitswelt als auch aus Sicht des freiwilligen Einsatzes im Privatleben hilfreich. Obwohl die Nutzungsentscheidung in der Arbeitswelt und im Privatleben zum Teil unterschiedlichen Rahmenbedingungen unterliegt, lassen sich übergeordnete Kategorien bilden, auf die sich die jeweils relevanten Einflussfaktoren zurück führen lassen.

- Ausgewogenheit der anwendungsspezifischen Betrachtung: Die Auswahl und Ausgewogenheit der akzeptanzrelevanten Einflussfaktoren spielen eine wichtige Rolle, da erst diese die differenzierte Betrachtung der Benutzerakzeptanz ermöglichen. Die grundlegende Methodik eines Akzeptanzmodells muss die Festlegung einer ausgewogenen Menge von wesentlichen, individuell messbaren und voneinander unabhängigen Akzeptanzindikatoren gewährleisten.

Es wird hier unterstellt, dass ein Akzeptanzmodell, das auf die beschriebenen Zielsetzungen hin konstruiert wird, einen Beitrag zur Entwicklung effektiver Anwendungen leistet. Der DART-Ansatz wurde erstmals in [Am $\left.{ }^{+} 02\right]$ vorgestellt. Dabei wurde die Umsetzung des DART-Ansatzes an zwei Anwendungen aus dem mobilfunkttechnischen Kontext aufgezeigt.

\section{Die Methodik des DART-Ansatzes}

Der Aufbau des DART-Ansatzes orientiert sich an der Grobstruktur der Balanced Scorecard (BSC) [KaNo96]. Diese stellt ein allgemein anerkanntes und vielfach eingesetztes Instrument zur Unternehmenssteuerung dar. Ihre Methodik sorgt für die Entwicklung eines ausgewogenen Kataloges von Kennzahlen und Messgrößen für die Unternehmenssteuerung. Umfassende Kategorien und Dimensionen stellen eine ausgewogene und vollständige Betrachtung aller relevanten Aspekte sicher. Diese Idee ist die Basis für den DART-Ansatz, um ebenfalls eine Vollständigkeit und Ausgewogenheit der verschiedenen Kriterien zur Analyse und Evaluierung der Benutzerakzeptanz zu gewährleisten. Aufgrund seiner empirischen Aussagekraft wird für den DART-Ansatz die Terminologie der empirischen Sozialforschung übernommen. Deshalb werden die Einflussfaktoren auf die Benutzerakzeptanz im Folgenden mit Indikatoren bezeichnet [May02].

Im Folgenden wird der DART-Ansatz in seinem dreigeteilten Detaillierungsgrad beschrieben. Zunächst erfolgt eine Erläuterung seiner Metastruktur, die aus vier Kategorien besteht. Anschließend werden vier Dimensionen eingeführt, die sich in weitere Subdimensionen verfeinern lassen.

Die Metastruktur des DART-Ansatzes setzt sich aus einer 2x2-Matrix von komplementären und orthogonalen Kategorien zusammen, mit Hilfe derer die Entwicklung von überschneidungsfreien und abgrenzungsscharfen Akzeptanzdimensionen gewährleistet werden soll: 
- Nutzen und Aufwand: Wir differenzieren zwei Kategorien, welche die Gesamtheit aller positiv unterstützenden und negativ verstärkenden Indikatoren auf die Benutzerakzeptanz erfassen.

- Innovative Anwendung und Kontextuelle Bedingungen der Innovation: Während die Kategorie Innovative Anwendung produktspezifische Akzeptanzfaktoren betrachtet, finden sich in der dazu komplementären Kategorie Kontextuelle Bedingungen der Innovation die kulturellen, sozialen und wirtschaftlichen Rahmenbedingungen wieder, die bei Akzeptanzentscheidungen von innovativen Technologien eine große Rolle spielen [Harn ${ }^{+} 99$, Herr $\left.{ }^{+} 99\right]$ ].

Die komplementäre Einteilung Nutzen und Aufwand ist in vielen Modellen zur Akzeptanzmessung als grundlegende Abwägung in kognitiven Entscheidungsprozessen bestätigt [Dav89, Dege86, Good95]. Die Betrachtung der kontextuellen Bedingungen der Innovation findet sich bereits in [Dege86]. Abbildung 1 stellt die Kategorien und die nachfolgend erläuterten Dimensionen des DART-Ansatzes vor:

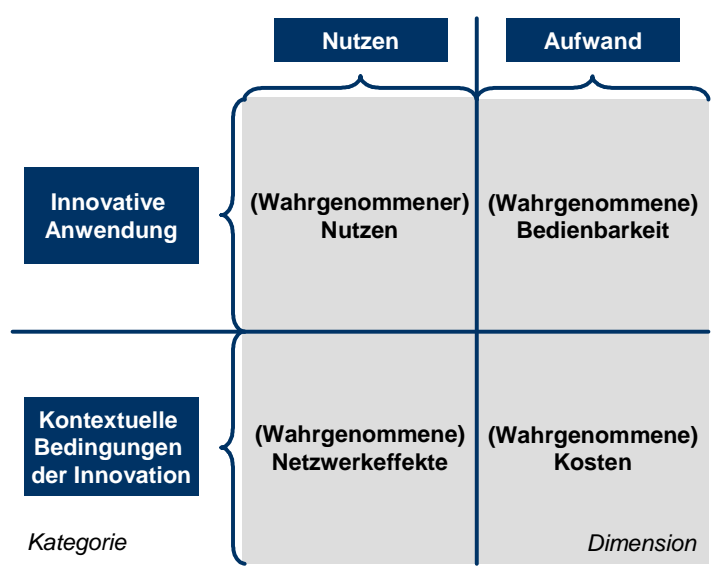

Abbildung 1: Die Kategorien und Dimensionen des DART-Ansatzes

Aus der vorgestellten Metastruktur leiten sich vier Dimensionen zur Betrachtung und Beschreibung der Akzeptanz ab: Nutzen, Bedienbarkeit, Netzwerkeffekte und Kosten.

- (Wahrgenommener) Nutzen: Die Dimension, die von den Kategorien Nutzen und Innovative Anwendung gebildet wird, beschreibt direkt den subjektiv empfundenen Nutzen des Benutzers. Exemplarische Indikatoren zur Messung dieser Akzeptanzdimension sind die Erwartungskonformität, das Informationsangebot, etc.

- (Wahrgenommene) Bedienbarkeit: Der Quadrant, welcher durch die Kategorien Aufwand und Innovative Anwendung charakterisiert ist, wird mit der Dimension der (Wahrgenommenen) Bedienbarkeit identifiziert. Die Bedienbarkeit wird in diesem Zusammenhang als subjektiv empfundener Aufwand in- 
terpretiert, der zur Verwendung einer Applikation überwunden werden muss und in der Regel keinen eigenen Anreiz zur Nutzung einer Applikation darstellt. Indikatoren zur Messung dieser Akzeptanzdimension sind beispielsweise bei einer Software die Navigation und Menüführung, das Design der Benutzeroberfläche, die Einfachheit und Intuition der Bedienung.

- (Wahrgenommene) Netzwerkeffekte: Die Kategorien Nutzen und Kontextuelle Bedingungen der Innovation bilden die Dimension (Wahrgenommene) Netzwerkeffekte. Die Betrachtung von Netzwerkeffekten erscheint im Zusammenhang mit innovativen Anwendungen als wesentlich, da ihr Nutzen abgesehen von den eigentlichen Produkteigenschaften in einem hohen Maße von der kulturellen, sozialen und wirtschaftlichen Verbreitung abhängig ist. Exemplarische Messgrößen und Indikatoren für diese Akzeptanzdimension sind Prestigewert einer Anwendung und Infrastruktur (Netzabdeckung eines Mobilfunknetzes).

- (Wahrgenommene) Kosten: Die Dimension (Wahrgenommene) Kosten wird durch die Kategorien Aufwand und Kontextuelle Bedingungen der Innovation gebildet. Oftmals bewirken innovative Anwendungen tief greifende Veränderungen in technologischen Prozessen und sozialen Systemen, die monetäre wie auch nicht-monetäre Kosten verursachen. Dazu zählen beispielsweise Anschaffungskosten, laufende Kosten, Kostentransparenz, gesundheitliche Risiken, Datenschutz, etc.

Die vier Dimensionen zeichnen sich insbesondere durch das Konstrukt der Wahrgenommenheit aus. Dieses soll verdeutlichen, dass die Angaben und Einschätzungen der befragten (potenziellen) Benutzer ausschließlich von ihrer Sichtweise und ihrer empfundenen Subjektivität abhängig sind. Die Dimensionen (Wahrgenommener) Nutzen und (Wahrgenommene) Bedienbarkeit finden sich bereits im Technologieakzeptanzmodell (TAM) wieder [Dav86, Dav89, $\operatorname{Dav}^{+}$89]. Der metastrukturale Aufbau des DART-Ansatzes verdeutlicht, dass TAM sich nur auf die Eigenschaften der innovativen Anwendung bezieht und die kontextuellen Bedingungen gegebenenfalls indirekt betrachtet. Diese Ausrichtung ist für die umfassende und ausgewogene Analyse und Evaluierung der Benutzerakzeptanz innovativer Anwendungen jedoch nicht ausreichend. Akzeptanzentscheidungen sind in einem hohen Maße von den umgebenden Rahmenbedingungen abhängig und werden in einigen Akzeptanzmodellen explizit berücksichtigt [Dege86, HeMo99]. Die Kategorie der Kontextuellen Bedingungen der Innovation umfasst genau diese ausgedehnte Betrachtungsweise. Damit bildet der DART-Ansatz ein Modell, das den inhaltlichen Zielen der Vollständigkeit und Ausgewogenheit entspricht.

In der Regel ist eine weitere Untergliederung der Dimensionen für eine ausgewogene Aufstellung von Akzeptanzindikatoren hilfreich. Dabei stellt die Akzeptanzbildung eine wichtige Grundlage dar. Eine Verfeinerung in die Subdimensionen Einstellungsakzeptanz und Nutzungsakzeptanz [vgl. Koll98, Koll99a] hilft, die Diskrepanz zwischen einer theoretischen Meinung zu einem Produkt und einem 
tatsächlichen Nutzungsverhalten zu schließen. Beide Akzeptanzphasen werden für die ausgewogene Betrachtung der Benutzerakzeptanz benötigt. Von der Integration der Handlungsakzeptanz wurde bei der Entwicklung des DART-Ansatzes abgesehen, da diese Phase der Akzeptanzbildung nicht als entscheidend erkannt werden kann. Zwei Beispiele sollen diese Meinung verdeutlichen. Die Handlungsakzeptanz kann nicht als Teil der Akzeptanzbildung angesehen werden, wenn ein Mitarbeiter im unternehmerischen Umfeld zur Nutzung einer innovativen Technologie angehalten wird. Die Entscheidung zum Kauf und zum Einsatz eines innovativen Produktes wird in diesem Fall von einer anderen Person als dem Benutzer, in der Regel von dem Vorgesetzten oder dem Leiter der technischen Abteilung, gefällt. Des Weiteren zeigte sich in den letzten Jahren eine steigende Tendenz zum Verschenken innovativer Produkte. So verzeichnete das Weihnachtsgeschäft der letzten Jahre einen starken Trend zum Verschenken eines Mobiltelefons. Da auch in diesem Fall die selbstständige Entscheidung des Benutzers für den Kauf eines innovativen Produktes fehlt, wurde zum Zweck der allgemeinen Anwendung des DART-Ansatzes von dieser Phase abgesehen. Weitere alternative oder ergänzende Gliederungskriterien wie beispielsweise eine Untergliederung in Emotional und Rational sind denkbar. Dies wird im Folgenden jedoch nicht weiter vertieft.

Die vorgestellten Dimensionen bilden einen allgemeinen Rahmen für die Spezifikation von Akzeptanzindikatoren einer innovativen Anwendung. In Anlehnung an die Balanced Scorecard ist es das Ziel, möglichst wenige, gegebenenfalls aggregierte Indikatoren mit einer hohen Aussagekraft festzulegen. Die erarbeiteten Indikatoren müssen den Anforderungen Nachhaltigkeit, Messbarkeit, Erreichbarkeit, Nachvollziehbarkeit und Zeitnähe genügen. Das genaue Vorgehen zur Anwendung des DART-Ansatzes wird im folgenden Kapitel dargestellt.

\section{Das Vorgehen des DART-Ansatzes}

Der DART-Ansatz ist als übergreifendes Akzeptanzmodell entwickelt worden, dass verschiedenste Anwendungsbereiche von innovativen Technologien unterstützt. Die hergeleiteten Dimensionen und Kategorien bilden einen allgemeinen Rahmen für die Spezifikation von Akzeptanzindikatoren für konkrete Anwendungen. Im Gegensatz zu vielen anderen Akzeptanzmodellen gibt der DART-Ansatz nicht nur die allgemeine Methodik vor, sondern unterstützt außerdem die Phasen der Entwicklung der Akzeptanzindikatoren über die Messung der Akzeptanz bis hin zur Analyse und Visualisierung der Ergebnisse. Damit ist der DART-Ansatz weitreichender zu sehen als die bestehenden Akzeptanzmodelle, die kein explizites Vorgehen zur Entwicklung von spezifischen Indikatoren berücksichtigen. Mit dem Vorgehen verfolgen wir die Zielsetzung, einen vollständigen und geschlossenen Ansatz zu präsentieren, der zur Entwicklung, Analyse und Evaluierung der akzeptanzrelevanten Indikatoren ein geeignetes Instrumentarium nach betriebswirtschaftlichem Vorbild bereitstellt. 
Ein bewährtes Vorgehen zur Spezifikation von betriebswirtschaftlichen Entscheidungsgrößen und Kennziffern ist in dem Ansatz der Balanced Scorecard enthalten. Es werden kritische Messgrößen festgelegt, die in einem wiederkehrenden Prozess erhoben werden und stetig mit den Zielwerten verglichen werden. Ergeben sich Abweichungen, werden Aktionen und Maßnahmen eingeleitet, um das gewünschte Niveau zu erreichen oder erneut herzustellen [HoPa01]. Diese Vorgehensweise wird im angloamerikanischen Raum mit plan, do, act bezeichnet und als Grobeinteilung der einzelnen Schritte verwendet. Das grundlegende Vorgehen für die Verwendung des DART-Ansatzes stellt Abbildung 2 in einer Übersicht vor:

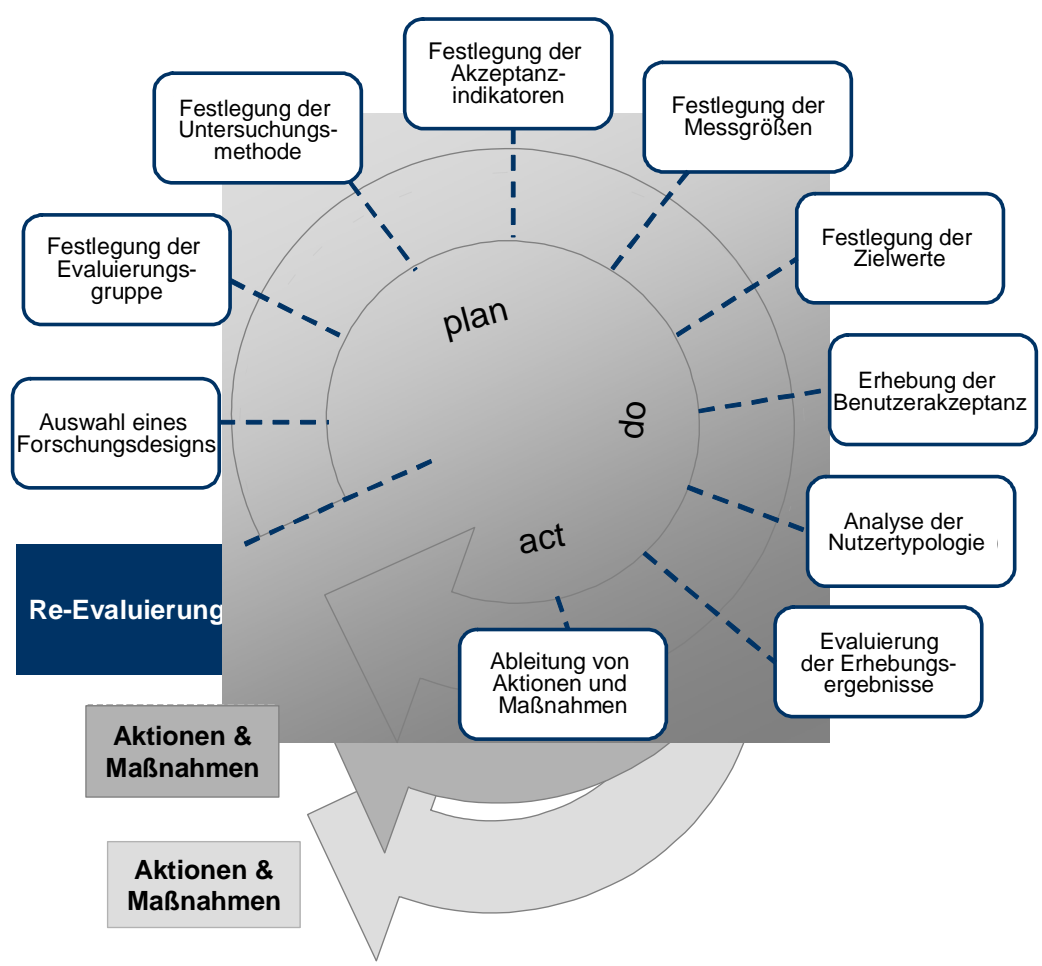

Abbildung 2: Das Vorgehen zur Anwendung des DART-Ansatzes

Der DART-Ansatz ist ein Instrument zur kontinuierlichen Messung und Evaluierung akzeptanzrelevanter Indikatoren. Die wiederkehrende Abfolge von Schritten zur Erhebung und Bewertung der Akzeptanz wird als Re-Evaluierung bezeichnet. Der Zyklus wird grob in drei Phasen unterteilt: Die Planungsphase (plan), die Ausführungsphase (do) und die Aktionsphase (act). Der Zyklus der ReEvaluierung beginnt mit der Planungsphase.

\section{Die Planungsphase umfasst folgende Schritte:}

- Auswahl eines Forschungsdesigns: Die Auswahl des Forschungsdesigns legt die Gesamtheit aller Entscheidungen ,wer, was, mit welchen Methoden, wann 
und wie oft erhebt" fest [May02]. Da sich die Messung von Akzeptanzindikatoren im Vergleich zu der Messung der Unternehmenskennzahlen der Balanced Scorecard im sozialwissenschaftlichen Bereich bewegt, sind grundlegende Überlegungen über das Design zu Beginn einer Akzeptanzuntersuchung notwendig.

- Festlegung der Evaluierungsgruppe: Um ein repräsentatives Ergebnis zu gewinnen, das dem Querschnitt der Zielgruppe entspricht, muss die Zusammensetzung der Evaluierungsgruppe genau geplant sein. Dabei reichen die klassischen Typologien, die auf soziodemografischen Merkmalen gründen, heutzutage nicht mehr aus $\left[\operatorname{Harn}^{+} 99,10\right]$. Vielmehr müssen zusätzlich weitere Indiktoren wie beispielsweise der Life-Style sowie die Struktur des sozialen Netzwerkes betrachtet werden.

- Festlegung der Untersuchungsmethode: Für die Erhebung der Benutzerakzeptanz stehen verschiedene Methoden zur Auswahl: Interview, schriftliche Befragung, Prototype-Testing, Teilnahme an einem Feldversuch bzw. Pilotprojekt usw. Es ist zweckmäßig, sich schon zu Beginn über die zur Verfügung stehenden Untersuchungsmethoden zu informieren und sich für die geeignete zu entscheiden, da diese wiederum die Wahl der Messgrößen für die einzelnen Akzeptanzindikatoren beeinflussen kann. Dabei bestimmt die Zielgruppe oftmals die Wahl der Untersuchungsmethode. So sind z.B. für Kinder grundsätzlich andere Untersuchungsmethoden geeignet als für Erwachsene.

- Festlegung der Akzeptanzindikatoren: Für die vier Dimensionen müssen Indikatoren gefunden werden, welche die Akzeptanz umfassend beschreiben. Überdies sind in jeder Dimension die Einstellungs- und Nutzungsakzeptanz ausgewogen zu betrachten. Zusätzlich oder alternativ wird die gleichwertige Berücksichtigung rationaler und emotionaler Indikatoren vorgeschlagen.

- Festlegung der Messgrößen: In dieser Phase werden die Akzeptanzindikatoren in Messgrößen überführt und jeweils mit Gewichtungsfaktoren und Messskalen abgerundet. Die Messgrößen müssen als zu bewertende Fragen bzw. Aussagen formuliert werden. Die Messgrößen können entweder qualitativ ermittelt oder auf einer diskreten Bewertungsskala aufgetragen werden.

- Festlegung der Zielwerte: Für die einzelnen Messgrößen werden vor der eigentlichen Akzeptanzerhebung Zielwerte festgelegt, um nach der Erhebung einen Vergleich zwischen den Zielwerten und den ermittelten Akzeptanzwerten zu ermöglichen.

Ein allgemeines Problem der sozialwissenschaftlichen Methodik ist die Erarbeitung interpretierbarer und zutreffender Indikatoren innerhalb der einzelnen Dimensionen für eine bestimmte Anwendung. Eine weitere Schwierigkeit besteht in der Überführung dieser Indikatoren in Messgrößen und Fragen. Gerade die Art der Formulierung kann erhebliche Auswirkungen auf die Ergebnisse haben. Für eine 
Betrachtung derartiger entscheidungskognitiver und psychologischer Aspekte muss auf spezielle Literatur verwiesen werden, z. B. [May02, Schu00].

Eine Überführung der qualitativen Ausprägungen in quantitative Größen ermöglicht eine detaillierte und aussagekräftige Analyse. Die Feinheit der Bewertungsabstufungen kann hierbei von einer dichotomen Ja/Nein-Entscheidung bis zu einer vielfältigen Abstufung der Akzeptanzentscheidung (Skala von 1-10 oder Skala von 1-6) reichen. So kann beispielsweise eine qualitative Ja/Nein-Bewertung als Binäruntersuchung gedeutet werden, indem 0 für eine nicht vorhandene Akzeptanzausprägung und 1 für eine maximale Ausprägung vergeben werden [Dege86]. Eine Dreiteilung der Skala in die Ausprägungen erfüllt/indifferent/nicht erfüllt kann ebenfalls in eine qualitative Messskala von 0 bis 1 überführt werden (vgl. Abbildung 3). Die Überführung in Messgrößen erlaubt bei der Datenauswertung weitreichende Analysemöglichkeiten wie eine Clusteranalyse zur Nutzertypenbestimmung.

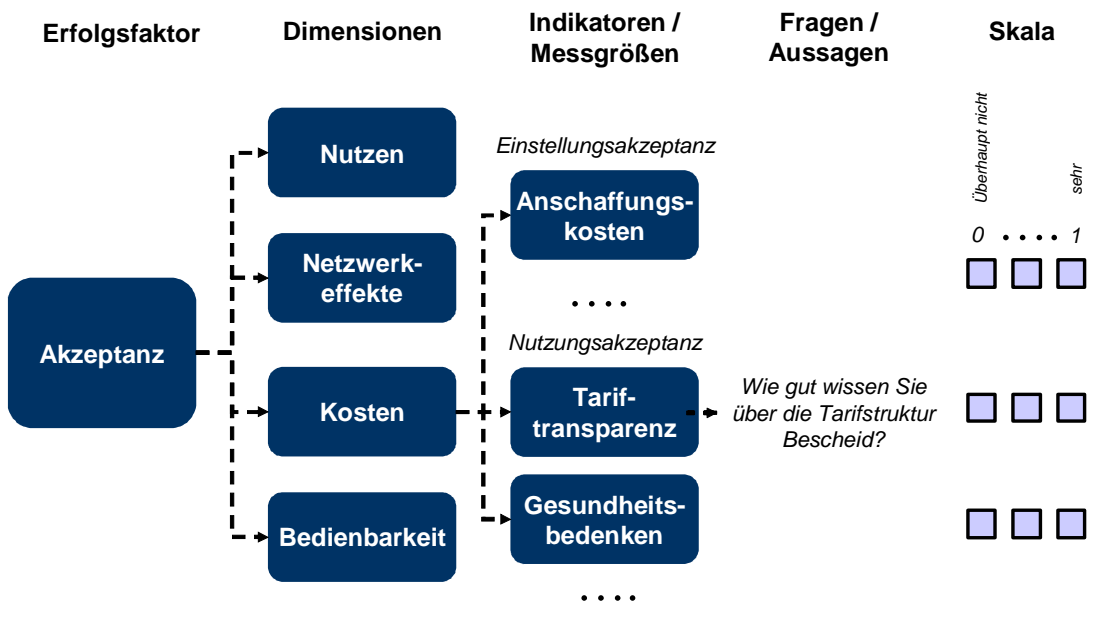

Abbildung 3: Operationalisierung der Akzeptanzdimensionen [vgl. May02]

Die stufenweise Gewichtung erlaubt es, mehrere Akzeptanzindikatoren innerhalb einer Dimension zu einem aggregierten Indikator zusammenzufassen. So stellt ein Indikator eine Zusammenfassung mehrerer aggregierter Messgrößen dar. Dahingegen werden einzelne Größen, die sich nicht zu einem Indikator aggregieren lassen, als Messgrößen bezeichnet.

Der Entwurf der Messgrößen wird mit einer Überprüfung der Konstruktvalidität abgeschlossen. Diese kann in der Analysephase über die Interkorrelation der Indizes verifiziert werden: Treten genügend hohe Korrelationen der Messgrößen eines Akzeptanzindikators auf, können diese als Beweis der Konstruktvalidität interpretiert werden [Dege86]. 
Die Planungsphase stellt den umfassendsten Anteil des Re-Evaluierungszyklus dar. An die Planungsphase schließt sich die Ausführungsphase an.

\section{Zur Ausführungsphase gehören folgende Schritte:}

- Erhebung der Benutzerakzeptanz: Nach der Festlegung der Akzeptanzindikatoren und ihrer Messgrößen wird die eigentliche Erhebung als empirische Untersuchung durchgeführt. Hilfreich in diesem Zusammenhang sind Ausführhinweise für die Befragten, um die Subjektivität bei der Bewertung zu objektivieren.

- Analyse der Nutzertypologie: Oftmals können Nutzertypen identifiziert werden, die ähnliche Akzeptanzentscheidungen treffen. In diesem Zusammenhang gibt eine Clusteranalyse der Erhebungsergebnisse darüber Auskunft, ob sich eine Typologie der Benutzer definieren lässt bzw. ob die Zielgruppe sich in bestimmte Nutzergruppen mit unterschiedlichem Anwendungsfokus aufteilen lässt.

- Evaluierung der Erhebungsergebnisse: Bei der Evaluierung werden die aufbereiteten Daten der Erhebung (Ist-Werte) mit den Zielvorstellungen (SollWerte) verglichen. Die Ergebnisse können in einem Spiderchart visualisiert werden (vgl. Abbildung 5).

Bei der Analyse der Nutzertypologie ist darauf zu achten, dass die Benutzer bis zum Zeitpunkt der Messung ausreichende Erfahrungen sammeln konnten, um differenzierte Nutzungsmuster auszubilden. Statistisch betrachtet lassen sich Nutzertypen erst dann feststellen, wenn das Rauschen der Basisnutzung soweit unterdrückt werden kann, dass Besonderheiten der Nutzung sichtbar werden [Dege86]. Dies macht sich meistens dadurch bemerkbar, dass der durchschnittliche Abstand der Gruppenmitglieder zu ihrem Clusterzentroid im Vergleich zu den Abständen der Clusterzentroide zueinander sehr groß ist. Die mathematische Konsequenz ist, dass die Standardabweichungen der Variablen innerhalb eines Clusters zu groß und die Strukturiertheit der Distanzmatrix zu gering ist, als dass eine angemessene Trennung von Benutzertypen erreicht werden kann. Im Allgemeinen sind derartige Analysen erst ab einer genügend großen Grundgesamtheit durchführbar.

Die gemittelten Ergebnisse der Erhebung werden für die einzelnen Akzeptanzindikatoren auf einer Skala aufgetragen. Zusätzlich kann die Streuung visualisiert werden, indem Ausprägungsbalken die minimale und die maximale Ausprägung um den Mittelwert veranschaulichen (vgl. Abbildung 4). 


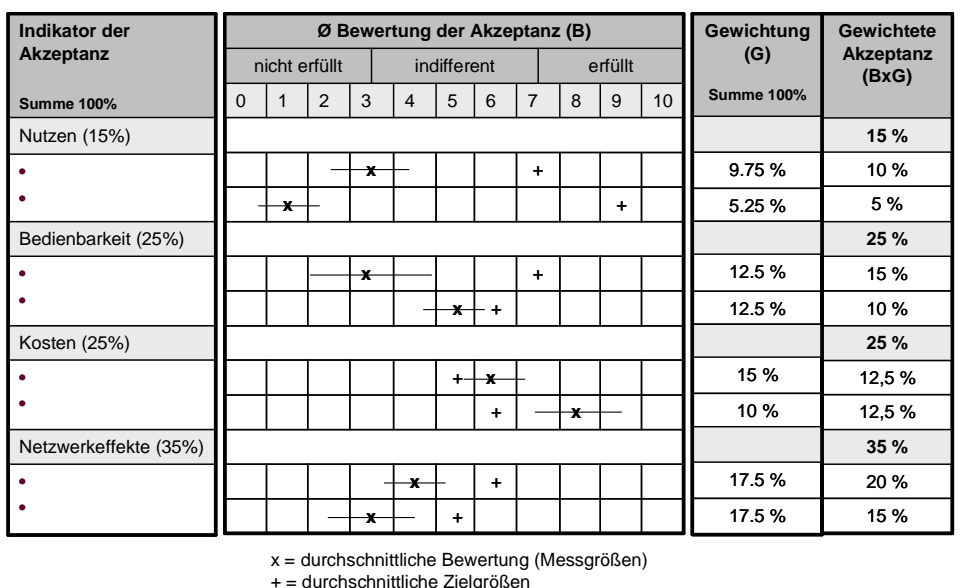

Abbildung 4: Auswertungsmatrix

Für die Dimensionen können Gewichtungsfaktoren eingeführt werden, damit eine flexible Anwendung entsprechend der Ansprüche und Einschätzungen der Benutzer gewährleistet wird. Die mittlere Ausprägung der Messgrößen multipliziert mit den Gewichtungsfaktoren kann als prozentualer Beitrag zu einem Indikator verstanden werden. Analog lässt sich die Summe der Indikatoren als mittlere prozentuale Akzeptanz einer Dimension interpretieren. Aus dem schematischen Beispiel in Abbildung 4 ist somit zu folgern, dass die Dimension Bedienbarkeit zu $25 \%$ erfüllt ist.

Abbildung 5 stellt die Visualisierung der Indikatoren im DART-Ansatz in Form eines Spiderchart vor, dessen symmetrische Anordnung an eine Dartscheibe erinnert. 


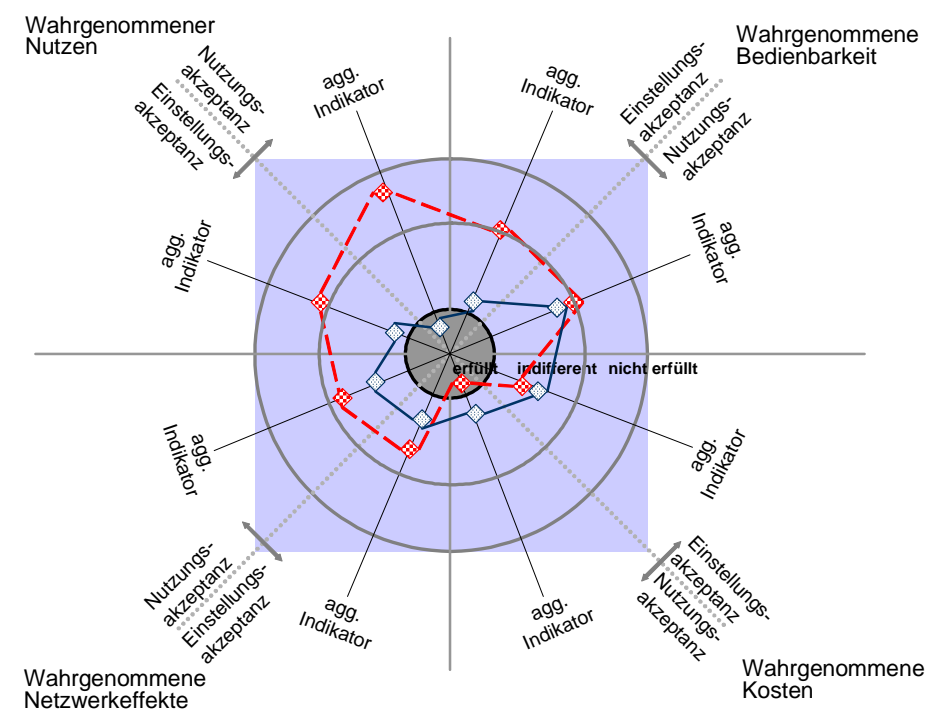

Abbildung 5: Visualisierungsmethodik des DART-Ansatzes

Die vier Dimensionen wurden jeweils in die Subdimensionen Einstellungs- und Nutzungsakzeptanz unterteilt. Daraus resultieren acht Bereiche, in denen die zu bewertenden Indikatoren sternförmig aufgetragen werden. Die Skalenorientierung der Indikatoren ist so anzuordnen, dass die positiven Ausprägungen nahe des Zentrums liegen. Für die Skalierung gilt es, die Messbereiche grafisch gleich zu verteilen. So lassen sich beispielsweise drei Messbereiche in erfüllt (positiv), indifferent und nicht erfüllt (negativ) einteilen.

\section{Die Aktionsphase bildet den Abschluss eines Zyklus zur Re-Evaluierung:}

Die im Soll-Ist-Vergleich gewonnenen Erkenntnisse dienen als Basis zur Ableitung von Maßnahmen und Aktionen, um die Diskrepanz zwischen Zielvorstellung (Soll-Zustand) und Realität (Ist-Zustand) zu beheben (Bestimmung der Aktionen). Eine generelle Aussage zum Ausmaß und zur Anzahl der Maßnahmen und Aktionen ist nicht möglich. Vielmehr hängt es von den Indikatoren und ihrer Ergebnisse $\mathrm{ab}$, ob je Indikator eine Maßnahme ausreichend ist oder mehrere Aktionen gebündelt eingeleitet werden müssen, um die Benutzerakzeptanz zu steigern. Beispiele für Maßnahmen und Aktionen sind die Erhebung der Kundenbedürfnisse, die Verbesserung der Ergonomie, die Abstimmung des Geschäftsmodells oder die Bildung strategischer Allianzen.

Für den stetigen Einsatz des DART-Ansatzes ist es hilfreich, Indikatoren zu finden, die über eine lange Zeit nutzbar und aussagekräftig bleiben. Wird dabei auf die konstante und unveränderte Verwendung der Indikatoren geachtet, kann ein genauer und zuverlässiger Vergleich erfolgen. Die Güte der Erkenntnisse ist außerdem von der Art der betrachteten Anwendung und dem Zeitpunkt der Betrachtung 
abhängig. So lassen sich konkrete Anwendungen sehr viel einfacher bewerten als abstrakte und visionäre Vorstellungen zukünftiger Produkte und Anwendungen. Demnach ist eine Analyse der Benutzerakzeptanz in den späten Entwicklungsphasen, die beispielsweise lauffähige Prototypen bereit stellen, einfacher als in den frühen Entwicklungsphasen, die sich durch unspezifische Produktbeschreibungen kennzeichnen. Es liegt prinzipiell im Ermessen eines Entscheidungsträgers der Produkt- oder Anwendungsentwicklung, wie oft und in welchen Phasen des Entwicklungsprozesses die Benutzerakzeptanz hinterfragt werden soll.

\section{Zusammenfassung}

Die Akzeptanzforschung stellt eine Vielzahl unterschiedlicher Akzeptanzmodelle bereit, mit denen aus diversen Sichtweisen die Benutzerakzeptanz erklärt werden kann. Doch mangelt es an Beiträgen, die mit ihren gewonnenen Erkenntnissen stetig sowohl einen analysierenden als auch einen gestaltenden Einfluss im Sinne einer Produktverbesserung nehmen.

Mit dem DART-Ansatz wird ein Instrumentarium zur Analyse und Evaluierung der Benutzerakzeptanz innovativer Anwendungen angeboten. Sein Aufbau orientiert sich an der Grundidee der Balanced Scorecard und berücksichtigt die Dimensionen (wahrgenommener) Nutzen, (wahrgenommene) Bedienbarkeit, (wahrgenommene) Netzwerkeffekte und (wahrgenommene) Kosten. Sein methodisches Vorgehen erleichtert die Identifikation und Determinierung relevanter Indikatoren, die auf die Benutzerakzeptanz beeinflussend einwirken. Die ausgewogene Betrachtung der akzeptanzrelevanten Indikatoren erlaubt eine differenzierte Evaluierung der Benutzerakzeptanz. Der DART-Ansatz dient in erster Linie dazu, folgende inhaltlichen Erkenntnisse zu generieren:

- Identifikation und Determinierung der akzeptanzrelevanten Indikatoren

- Messung, Analyse und Evaluierung der Benutzerakzeptanz

- Identifikation und Durchführung von geeigneten gestaltenden Maßnahmen und Aktionen, die sich positiv auf die Benutzerakzeptanz auswirken

- Messung des Erfolgs und des Fortschritts

Die Anwendung des DART-Ansatzes ist sowohl im Produktentwicklungszyklus als auch im Produktlebenszyklus hilfreich. Die Ableitung gestaltenden Maßnahmen erlaubt in beiden Fällen das gezielte Eingreifen, um ein Produkt zu verbessern und seine Diffusionschance zu vergrößern. Damit wird deutlich, dass der DARTAnsatz trotz seiner Fokussierung auf innovative Anwendungen auch bei nichtinnovativen Anwendungen und Produkten zum Einsatz kommen kann. Aufgrund seiner allgemeinen Gültigkeit lässt sich seine praktische Umsetzung nicht auf eine bestimmte Kategorie von Gütern reduzieren. 
Es wurde deutlich, dass der DART-Ansatz eine stetige und wiederkehrende Anwendung ermöglicht. Somit leistet er einen wertvollen Beitrag, um den Fortschritt der (Weiter-) Entwicklung einer innovativen Anwendung erkennbar zu machen. Nur bei der erstmaligen Anwendung des DART-Ansatzes entsteht ein großer Aufwand, da die umfangreiche Planungsphase in detaillierter und korrekter Art und Weise durchgeführt werden muss. Die stetige Verwendung des DART-Ansatzes geht dahingegen mit einem deutlich geringeren Aufwand einher, weil sich die notwendigen Tätigkeiten auf die Ausführungsphase und Aktionsphase fokussieren.

Die praktische Umsetzung des DART-Ansatzes wurde an diversen Anwendungen vor allem im mobilfunkttechnischen Kontext verifiziert. Dazu gehören mobile Anwendungen wie Short Message Service (SMS) und innovative Anwendungen wie beispielsweise multimediales Kinoticketing [Am+02]. Die praktische Umsetzung auf eine innovative Anwendung in der Arbeitswelt ist derzeit in Vorbereitung.

\section{Literatur}

[Am+02] Amberg, M., Hirschmeier, M., Wehrmann, J.: The Compass Acceptance Model for the Analysis and Evaluation of Mobile Information Systems, International Journal of Mobile Communications (IJMC), 2002.

[Dav86] Davis, F. D.: A Technology Acceptance Model for Empirically Testing New EndUser Information Systems: Theory and Results, in MIT Sloan School of Management. Cambridge, 1986.

[Dav89] Davis, F. D.: Perceived Usefulness, Perceived Ease of Use, and User Acceptance of Information Technology, MIS Quarterly, pp. 319-339, 1989.

[Dav $\left.{ }^{+} 89\right]$ Davis, F. D.; Bagozzi, R. P.; Warshaw, P. R: User acceptance of computer technology: A comparison of two theoretical models. In: Management Science. 35 (1989) 8, pp. 982-1003, 1989.

[Dege86] Degenhardt, W.: Akzeptanzforschung zu Bildschirmtext: Methoden und Ergebnisse. München, 1986.

[Döh183] Döhl, W.: Akzeptanz innovativer Technologien in Büro und Verwaltung. Göttingen, 1983.

[Good95] Goodhue, D. L.: Understanding User Evaluations of Informations Systems. In: Management Science, 41, 1827-1844, 1995.

[Good95] Goodhue, D. L.; Thompson, R.L.: Task-Technology Fit and Individual Performance, MIS Quarterly, 19:2 (6/1995), pp. 213-236, 1995.

[Harn $\left.{ }^{+} 99\right]$ Harnischfeger, M., Kolo, C., Zoche, P.: Elemente eines Akzeptanzmodells. In: Szyperski, N. (Hrsg) (1999): Perspektiven der Medienwirtschaft. Köln, 1999. 
[Herr99] Herrmann, T.: Perspektiven der Medienwirtschaft. Kompetenz - Akzeptanz - Geschäftsfelder. In: Szyperski, N. (Hrsg.): Perspektiven der Medienwirtschaft. Köln, 1999.

[HoPa01] Horvath \& Partner: Balanced Scorecard umsetzen. Stuttgart 2001.

[HuC094] Hufnagel, E. M., Conca, C.: Use Response Data: The Potential for Errors and Biases, Information Systems Research, 5, pp.48-73, 1994.

[KaNo96] Kaplan, R.S.; Norton, D.P.: The Balanced Scorecard - Translating Strategy into Action. Boston, 1996.

[Kol198] Kollmann, T.: Akzeptanz innovativer Nutzungsgüter und -systeme : Konsequenzen für die Einführung von Telekommunikations- und Multimediasystemen. Wiesbaden, 1998.

[Kol199a] Kollmann, T.: Akzeptanzprobleme neuer Technologien - Die Notwendigkeit eines dynamischen Untersuchungsansatzes. In: Bliemel, F., Fassott, G., Theobald, A. (Hrsg.): Electronic Commerce. Wiesbaden, 1999.

[Koll99b] Kollmann, T.: Das Konstrukt der Akzeptanz im Marketing. In: WiSt Heft 3, 1999

[May02] Mayer, H. O.: Interview und schriftliche Befragung: Entwicklung, Durchführung und Auswertung. Oldenbourg, 2002.

[Me190] Melone, N. P.: A Theoretical Assessment of the User Satisfaction Construct in Information Systems Research, Management Science, 36, pp. 192-222, 1990.

[Nei90] Neibecker, B.: Werbewirkungsanalyse mit Expertensystemen. Heidelberg, 1990.

[Schr01] Schröder, R.: Zur Akzeptanz innovativer Verkehrsinformationssysteme: eine empirische Analyse im Rahmen des Telematik-Projektes BAYERININFO. Frankfurt/M., 2001.

[Sim01] Simon, B.: Wissensmedien im Bildungssektor - Eine Akzeptanzuntersuchung an Hochschulen, Dissertation. Wirtschaftsuniversität. Wien, 2001.

[Schu00] Schuhmann, S.: Repräsentative Umfrage: praxisorientierte Einführung in empirische Methoden und statistische Analyseverfahren. München, 2000. 